\title{
Development of a Non-invasive Assessment of Hypoxia and Neovascularization with Magnetic Resonance Imaging in Benign and Malignant Breast Tumors: Initial Results
}

\author{
Andreas Stadlbauer, ${ }^{1,2}$ Max Zimmermann, ${ }^{2}$ Barbara Bennani-Baiti, ${ }^{3}$ \\ Thomas H. Helbich, ${ }^{3}$ Pascal Baltzer, ${ }^{3}$ Paola Clauser, ${ }^{3}$ Panagiotis Kapetas, ${ }^{3}$ \\ Zsuzsanna Bago-Horvath, ${ }^{4}$ Katja Pinker $\oplus^{3,5}$ \\ ${ }^{1}$ Institute of Medical Radiology, University Clinic of St. Pölten, Propst-Führer-Straße 4, St. Pölten, 3100, Austria \\ ${ }^{2}$ Department of Neurosurgery, University of Erlangen-Nürnberg, Schwabachanlage 6, Erlangen, 91054, Germany \\ ${ }^{3}$ Department of Biomedical Imaging and Image-guided Therapy, Division of Molecular and Gender Imaging, Medical University of Vienna, \\ Waehringer Guertel 18-20, 1090, Vienna, Austria \\ ${ }^{4}$ Department of Pathology, Medical University of Vienna, Weahringer Guertel 18-20, Vienna, 1090, Austria \\ ${ }^{5}$ Department of Radiology, Breast Imaging Service, Memorial Sloan Kettering Cancer Center, 300 E 66th St, New York, NY, 10065, USA
}

\begin{abstract}
Purpose: To develop a novel magnetic resonance imaging (MRI) approach for the noninvasive assessment of hypoxia and neovascularization in breast tumors.

Procedures: In this IRB-approved prospective study, 20 patients with suspicious breast lesions (BI-RADS 4/5) underwent multiparametric breast MRI including quantitative BOLD (qBOLD) and vascular architecture mapping (VAM). Custom-made in-house MatLab software was used for qBOLD and VAM data postprocessing and calculation of quantitative MRI biomarker maps of oxygen extraction fraction (OEF), metabolic rate of oxygen $\left(\mathrm{MRO}_{2}\right)$, and mitochondrial oxygen tension $\left(\mathrm{mitoPO}_{2}\right)$ to measure tissue hypoxia and neovascularization including vascular architecture including microvessel radius (VSI), density (MVD), and type (MTI). Histopathology was used as standard of reference. Appropriate statistics were performed to assess and compare correlations between MRI biomarkers for hypoxia and neovascularization.

Results: qBOLD and VAM data with good quality were obtained from all patients with 13 invasive ductal carcinoma (IDC) and 7 benign breast tumors with a lesion diameter of at least $10 \mathrm{~mm}$ in all spatial directions. MRI biomarker maps of oxygen metabolism and neovascularization demonstrated intratumoral spatial heterogeneity with a broad range of biomarker values. Bulk tumor neovasculature consisted of draining venous microvasculature with slow flowing blood. High OEF and low mitoPO $\mathrm{O}_{2}$ were associated with low MVD and vice versa. The heterogeneous pattern of $\mathrm{MRO}_{2}$ values showed spatial congruence with VSI. IDCs showed significantly higher $\mathrm{MRO}_{2}(P=0.007)$, lower mitoPO $2(P=0.021)$, higher MVD $(P=0.005)$, and lower (i.e., more pathologic) MTI $(P=0.001)$ compared with benign breast tumors. These results indicate that IDCs consume more oxygen and are more hypoxic and neovascularized than benign tumors.

Conclusions: We developed a novel MRI approach for the noninvasive assessment of hypoxia and neovascularization in benign and malignant breast tumors that can be easily integrated in a diagnostic MRI protocol and provides insight into intratumoral heterogeneity.
\end{abstract}


Key words: MRI, Imaging biomarkers, Tumor hypoxia, Oxygen metabolism, Neovascularization, Breast cancer

\section{Introduction}

Breast cancer $(\mathrm{BC})$ is a complex disease with remarkable intratumoral heterogeneity which leads to varied genetic, phenotypic, and behavioral characteristics; clinical presentations; and treatment responses [1-6]. Tumor hypoxia has been recognized as an important feature and a key driver of $\mathrm{BC}$ heterogeneity [7] that leads to the development of cell clones and an aggressive and treatment-resistant phenotype characterized by rapid progression and poor prognosis [8]. In addition, the presence of tumor hypoxia has a profound influence on the efficacy of cancer therapies and is a strong prognostic factor for disease progression, metastases, and survival [7, 9]. To survive and grow in hypoxic conditions, tumor cells co-opt adaptive mechanisms, with a key mechanism being tumor neovascularization [10]. Increased tumor neovascularization has been associated with poorer recurrence-free, cancer-specific, and overall survival, as well as failure to respond to chemotherapy [11-14].

It has to be noted that the distribution of hypoxia and induced neovascularization are not uniform within a tumor and significantly contribute to BC heterogeneity [8]. Therefore, tumor hypoxia and the induced neovascularization may provide powerful physiological stimuli that can be exploited as a tumor-specific condition, enabling the design of hypoxiabased imaging biomarkers and hypoxia-activated anti-cancer drugs. Nevertheless, to date, the assessment of tumor hypoxia, neovascularization, and consequently $\mathrm{BC}$ heterogeneity, and the ability to stratify patients based on the tumor's hypoxia status is limited in its clinical application due to invasiveness [15] and poor reproducibility of the technique [16, 17], high costs (positron emission tomography, PET) [18-20], or low spatial resolution (near-infrared spectroscopy, NIRS) [21-23].

New individual magnetic resonance imaging (MRI) techniques which allow a non-invasive, quantitative measurement of hypoxia as well as microvascular vessel caliber and architecture with hitherto unparalleled accuracy have been developed and pioneered in patients with brain cancers [24-26]. Advanced quantitative blood oxygenation leveldependent (qBOLD) imaging allows the absolute quantification of the tissue oxygen extraction fraction (OEF), the oxygen metabolic rate $\left(\mathrm{MRO}_{2}\right)$, and the mitochondrial oxygen tension $\left(\right.$ mitoPO $\left.{ }_{2}\right)$ [27]. Through these, calculation of the actual tissue oxygen tension and thereby a direct measurement of tumor hypoxia becomes possible [28]. Vascular architectural mapping (VAM) can measure and quantify microvascular vessel caliber and architecture, thus depicting the development of aberrant tumor vessels indicative of neovascularization and response to antiangiogenic therapy in brain cancer [29].
In breast cancer, a technique that not only depicts tumor hypoxia but also the induced neovascularization and $\mathrm{BC}$ heterogeneity is necessary yet currently does not exist. We hypothesized that the non-invasive assessment of hypoxia and neovascularization with MRI is feasible in benign and malignant breast tumors and provides insights into intratumoral heterogeneity. The aim of this study was to develop a novel MRI approach for the non-invasive synergistic assessment of hypoxia and neovascularization in benign and malignant breast tumors, which can be integrated in a diagnostic MRI protocol.

\section{Material and Methods}

The institutional review board approved this prospective, single-institution study, and all patients gave written, informed consent.

\section{Patients}

Between January and March 2017, 20 consecutive female patients (mean age 49 years, range 21-84 years) who met the following inclusion criteria were enrolled and underwent multiparametric MRI of the breast at 3 Tesla: 18 years or older; not pregnant; not breastfeeding; suspicious imaging finding at mammography or breast ultrasound (Breast Imaging Reporting and Data System [BI-RADS] assessment category 4-5); lesion size $\geq 1 \mathrm{~cm}^{3}$; no previous treatment, i.e., breast biopsy before MRI or neoadjuvant chemotherapy; and no contraindications to MRI or MR contrast agents.

\section{MRI Data Acquisition}

All MRI examinations were performed on a 3 Tesla clinical scanner (Tim Trio, Siemens, Erlangen, Germany) equipped with a standard 16-channel breast coil (Sentinelle, Invivo, FL). In addition to a state-of-the-art MRI protocol [30] with T2-weighted, diffusion-weighted, and dynamic contrast enhanced (DCE) MR imaging, the following sequences were performed:

1. qBOLD imaging: (i) a multi-echo gradient echo (GE) sequence for T2*-mapping ( 8 echoes; TR: $750 \mathrm{~ms}$; TE: 5$40 \mathrm{~ms}$ ) and (ii) a multi-echo spin echo (SE) sequence for T2mapping (8 echoes; TR: $2000 \mathrm{~ms}$; TE: 15-120 ms).

2. VAM: (i) a separate DWI sequence ( $b$ values: 0 and $850 \mathrm{~s} / \mathrm{mm}^{2}$; TR/TE: 3000/53 ms; TA $50 \mathrm{~s}$ ) and (ii) a dynamic susceptibility contrast (DSC) bolus-tracking perfusion MRI sequence combined with a hybrid single-shot 
gradient echo spin echo (GESE) EPI readout [31] (TR: $1360 \mathrm{~ms}$; TE(GE): $25 \mathrm{~ms}$; TE(SE): $93 \mathrm{~ms}$ ).

Geometric parameters were identically chosen for the four qBOLD and VAM sequences: coronal slice orientation; field-of-view (FoV): $320 \times 240 \mathrm{~mm}$; in-plane resolution: $2.5 \times 2.5 \mathrm{~mm}$, slice thickness: $6 \mathrm{~mm}$; 8 slices. DSC GESE perfusion examinations were performed with 60 dynamic measurements during administration of a single dose (0.1 mmol/kg-bodyweight) of gadolinium-based contrast agents (GBCA; gadoterate-meglumine, Dotarem, Guerbet, France) at a rate of $4 \mathrm{ml} / \mathrm{s}$ followed by a $20-\mathrm{ml}$ bolus of saline using an MR-compatible injector (Spectris, Medrad). This resulted in 60 dynamic volumes of both GE-EPI and SE-EPI for tracking the first-pass peak contrast media bolus dynamics. The additional acquisition time (TA) for the qBOLD (R2* and R2-mapping: TA, 1.5 and $2.5 \mathrm{~min}$, respectively) and VAM sequences (DSC GESE perfusion and DW-EPI: TA, $1.4 \mathrm{~min}$ and $50 \mathrm{~s}$, respectively) was less than $7 \mathrm{~min}$.

Details of the MRI sequence protocol are shown in the top part of Fig. 1. In short, the diagnostic MRI sequences (T2w TSE, axial DWI, not shown in Fig. 1) were followed by the T2- and $\mathrm{T} 2 *$-mapping sequences for $\mathrm{qBOLD}$, the coronal DWI for VAM, and the native T1w FLASH sequence for DCE perfusion. Injection of contrast media was performed during DSC GESE perfusion sequence immediately followed by the five diagnostic dynamic $\mathrm{T} 1 \mathrm{w}$ FLASH sequences.

Hence, we obtained three different perfusion MRI data (GE-DSC, SE-DSC, and DCE perfusion) with injection of only one single dose of contrast media. Note: coronal slice orientation was chosen in order to (i) reduce FoV and motion artifacts (e.g., berating) compared to axial orientation and to (ii) facilitate AIF determination (i.e., using the inflow effect of the contrast media bolus and reducing blood flow parallel to the slices).

\section{MRI Data Processing and Calculation of MRI Biomarker}

Processing of qBOLD and VAM data as well as calculation of MRI biomarker maps for oxygen metabolism and neovascularization were performed with custom-made MatLab (MathWorks, Natick, MA) software.

The qBOLD data processing consisted of three steps: (i) corrections for background fields of the $\mathrm{T} 2 *$-mapping data [32] and for stimulated echos of the T2-mapping data [33]; (ii) calculation of relaxivity maps (R2* and $\mathrm{R} 2$ ) from the $\mathrm{T} 2 *_{\text {- }}$ and $\mathrm{T} 2$-mapping data; and (iii) calculation of absolute blood volume (BV) and flow (BF) maps from the GE data of the DSC GESE perfusion data via manual selection of a local arterial input function (AIF) [34] (Fig. 1, left part of "a MRI Data Acquisition") in GE-DSC by an experienced radiologist (K.P. or T.T.H.). These maps (R2*, R2, BV,
BF) were used for calculation of MRI biomarker maps of oxygen metabolism, including oxygen extraction fraction (OEF), metabolic rate of oxygen $\left(\mathrm{MRO}_{2}\right)$ [27], and the average mitochondrial oxygen tension $\left(\mathrm{mitoPO}_{2}\right)$ [35, 36], using the equations shown in Fig. 1, left part of panel "c Calculation of MRI biomarker" highlighted with "qBOLD". OEF describes the percent of the oxygen removed from the blood for tissue consumption. $\mathrm{MRO}_{2}$ is the rate of oxygen consumed by the tissue in $\mu \mathrm{mol} / 100 \mathrm{~g} \cdot$ minute, and $\mathrm{mitoPO}_{2}$ (in $\mathrm{mmHg}$ ) reflects the balance between the delivery and consumption of oxygen, i.e., the oxygen tension in the tissue.

The VAM data processing consisted of three steps: (i) normalization to the baseline (pre-bolus) signal which was determined as the mean of the signals from the 4th to the 15th dynamic volume, (ii) fitting of the first pass bolus curves for each voxel of the DSC GE and SE perfusion data with a previously described gamma-variate function [37], and (iii) calculation of the $\Delta \mathrm{R}_{2, \mathrm{GE}}$ versus $\left(\Delta \mathrm{R}_{2, \mathrm{SE}}\right)^{3 / 2}$ diagram [31], which is the so-called vascular hysteresis loop (VHL) (Fig. 1, right part of "b MRI Data Processing"). These data were used for calculation of MRI biomarker maps of neovascularization, including microvessel density (MVD; in units of $\mathrm{mm}^{-2}$ ), vessel size index (VSI, i.e., microvessel radius; in units of $\mu \mathrm{m}$ ) [38], and the microvessel type indicator (MTI; in units of $\mathrm{m}^{-5 / 2}$ ) [24]. Maps of MVD and VSI were calculated using the equations shown in Fig. 1, right part of panel "c Calculation of MRI biomarker" highlighted with "VAM". Calculation of the MTI map which was defined as the area of the vascular hysteresis loop (VHL) signed with the rotational direction of the VHL, i.e., a clockwise VHL direction, was identified with a plus sign, and a counter-clockwise VHL direction was identified with a minus sign. Based on the findings of Emblem et al. [39], if in a voxel of the VAM dataset in the vascular system venule- and capillary-like components are present, the VHL in this voxel transverses counterclockwise. If there are arterioles and capillaries, the VHL transverses clockwise.

\section{Quantitative Analysis}

For quantitative analysis, regions of interest (ROIs) were manually defined based on features seen in the MVD maps for high, medium, and low neovascularization. MRI biomarker values for oxygen metabolism (OEF, $\mathrm{MRO}_{2}$, mitoPO2) and for neovascularization (MVD, VSI, MTI) were averaged for each ROI. Additionally, the total tumor volumes were calculated on DCE-MR images.

\section{Histopathologic Analysis}

Histopathology was used as the standard of reference in all lesions by means of either image-guided needle biopsy or 


\section{a MRI Data Acquisition}

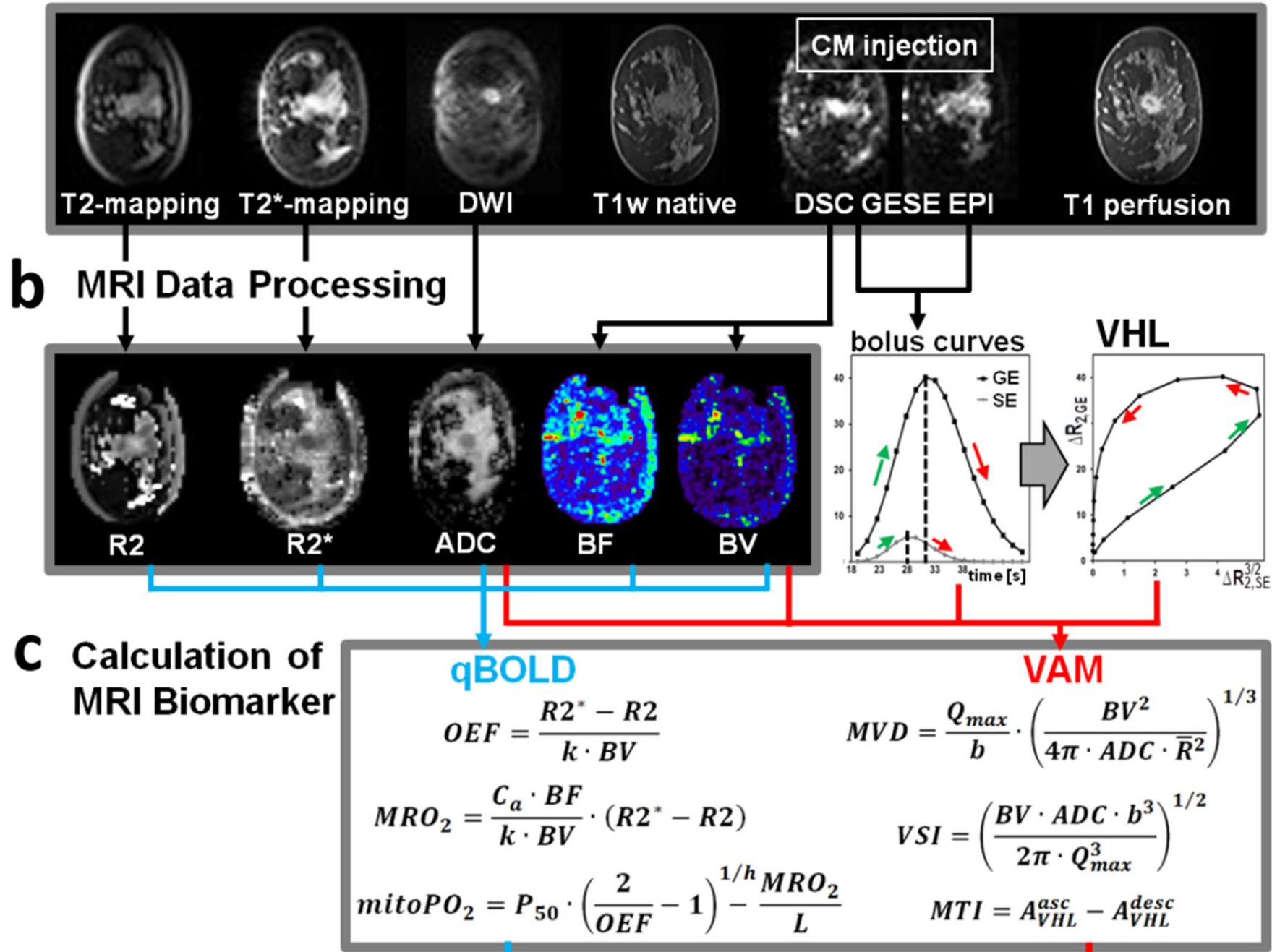

d Oxygen Metabolism

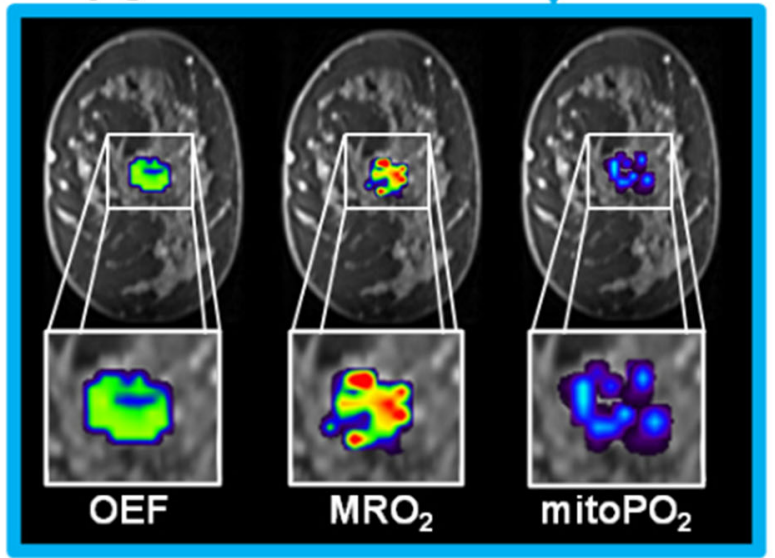

\section{Neovascularization}

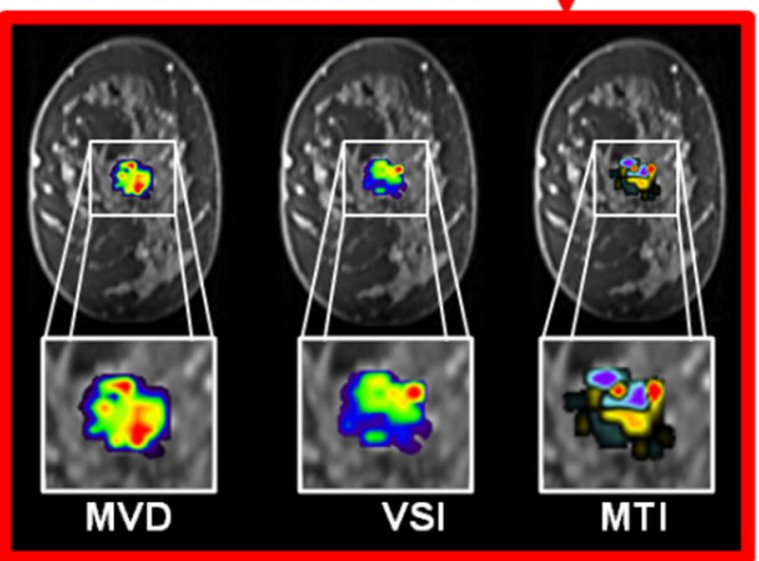

surgery, regardless of the imaging results. All cases were read by an experienced breast pathologist. For a benign diagnosis at image-guided needle biopsy, the final diagnosis was benign (seven of the 20 patients). For a high-risk lesion, which had an uncertain potential for malignancy, the final diagnosis was established with open surgery $(n=5)$. 
Fig. 1. MRI data processing pipeline for combined noninvasive in situ characterization of neovascularization, oxygen metabolism, and hypoxia, respectively, of human breast tumors. Firstly, an MRI data acquisition protocol was used which was adapted from neuroimaging research to be compatible with clinical routine breast imaging purposes. Secondly, data processing of MRI raw data included calculation of relativity maps ( $\mathrm{R} 2$ and $\left.\mathrm{R} 22^{*}\right)$, apparent diffusion coefficient (ADC), blood flow and volume (BF and BV), and vascular hysteresis loops (VHL), respectively. Finally, we used the equations shown by gray box for calculation of MRI biomarker maps of oxygen metabolism and hypoxia using the qBOLD approach and neovascularization using the VAM approach. Note: $k=4 / 3 \cdot \pi \cdot \gamma \cdot \Delta x \cdot \mathrm{Hct} \cdot \mathrm{B}_{0}(\gamma=$ $2.67502 \cdot 10^{8} \mathrm{rad} / \mathrm{s} / \mathrm{T}$ is the nuclear gyromagnetic ratio; $\Delta X=$ $0.264 \cdot 10^{-6}$ is the difference between the magnetic susceptibilities of fully oxygenated and fully deoxygenated hemoglobin; Hct $=0.42 \times 0.85$ is the microvascular hematocrit fraction); $C_{a}=8.68 \mathrm{mmol} / \mathrm{ml}$ is the arterial blood oxygen content; $P_{50}$ is the hemoglobin half-saturation tension of oxygen $(27 \mathrm{mmHg}), h$ is the Hill coefficient of oxygen binding to hemoglobin (2.7), and $L(4.4 \mu \mathrm{mol} / \mathrm{mmHg}$ per $\min )$ is the tissue oxygen conductivity; $Q_{\max }=\max \left[\Delta \mathrm{R}_{2, \mathrm{GE}}\right] /$ $\max \left[\left(\Delta \mathrm{R}_{2, \mathrm{GE}}\right)^{3 / 2}\right] ; \mathrm{R}^{-} \approx 3.0 \mu \mathrm{m}$ is the mean vessel lumen radius; and $b$ is a numerical constant $(b=1.6781)$. For guidance of the interpretation of MTI maps: negative MTI values were assigned to cool colors and positive to warm colors. Consequently, maps of MTI enabled differentiation between supplying arterial (areas with warm colors) and draining venous microvasculature (areas with cool colors). More specifically, a voxel with high-arterial blood volume, for example, shows a high positive MTI value and an orange to red color in the MTI maps; a capillary voxel shows a very low MTI value around 0 and a black color in the MTI maps; and a voxel with high venous blood volume shows a high, negative $\mathrm{MTI}$ value and a purple color in the MTI maps.

\section{Statistical Analysis}

Software (SPSS 21, IBM, Chicago, IL, USA) was used for statistical evaluation. A Mann-Whitney test was used for nonparametric comparison of metric biomarker values. Linear regression was performed and Spearman's rank correlation coefficient was calculated to investigate the association between MRI biomarkers for oxygen metabolism for hypoxia measurement and neovascularization. $P$ values less than 0.05 were considered to indicate significance.

\section{Results}

qBOLD and VAM data of good quality were obtained from 20 patients (mean age 47 years, range $21-84$ years). A total of 20 breast lesions were assessed. In 13 lesions, the histopathology revealed an invasive ductal carcinoma (IDC) and seven had a benign histological finding: five fibroadenoma (FA), one recurrent mastitis, and one intraductal papilloma. The mean lesion volume for these 20 patients was $9507 \mathrm{~mm}^{3}\left(881-34,523 \mathrm{~mm}^{3}\right)$ and the lesion diameter was at least $1 \mathrm{~cm}$ in all three directions of space. Details on patient and lesion characteristics are summarized in Table 1.

\section{Qualitative Analysis of MRI Biomarker Maps}

A representative case for MRI-based combined depiction of tumor hypoxia through quantitative assessment of oxygen metabolism and neovascularization in a patient with IDC is depicted in Fig. 2. MRI biomarker maps of both oxygen metabolism and neovascularization

Table 1. Patient and tumor characteristics

\begin{tabular}{|c|c|c|c|c|c|}
\hline ID & Age [years] & Histopathology & Location & Tumor volume $\left[\mathrm{mm}^{3}\right]$ & BI-RADS \\
\hline 1 & 39 & IDC & 11:00 axis, left breast & 5636 & $5 b$ \\
\hline 2 & 60 & IDC & 9:00 axis, left breast & 5817 & $5 b$ \\
\hline 3 & 48 & IDC & 4:00 axis, left breast & 1877 & $5 b$ \\
\hline 4 & 62 & intraductal papilloma & $8: 00$ axis, right breast & 919 & 3 \\
\hline 5 & 35 & IDC & $8: 00$ axis, right breast & 6004 & $5 b$ \\
\hline 6 & 48 & IDC & 3:00 axis, left breast & 3760 & $5 b$ \\
\hline 7 & 37 & IDC & $3: 00$ axis, left breast & 3940 & $5 b$ \\
\hline 8 & 21 & FA & 9:00 axis, left breast & 34,174 & 2 \\
\hline 9 & 56 & IDC & 9:00 axis, right breast & 8181 & $5 b$ \\
\hline 10 & 84 & IDC & 9:00 axis, left breast & 8508 & $5 \mathrm{~b}$ \\
\hline 11 & 50 & IDC & $3: 00$ axis, left breast & 10,325 & $5 b$ \\
\hline 12 & 58 & IDC & 3:00 axis, right breast & 2368 & $5 b$ \\
\hline 13 & 39 & FA & 2:00 axis, left breast & 1649 & 2 \\
\hline 14 & 53 & IDC & 4:00 axis, left breast & 11,289 & $5 b$ \\
\hline 15 & 53 & IDC & central, left breast & 15,482 & $5 \mathrm{~b}$ \\
\hline 16 & 31 & FA & $9: 00$ axis, right breast & 6589 & 2 \\
\hline 17 & 22 & FA & 2:00 axis, left breast & 31,656 & 2 \\
\hline 18 & 55 & recurrent mastitis & central, right breast & 20,473 & 2 \\
\hline 19 & 65 & IDC & 2:00 axis, right breast & 7592 & $5 b$ \\
\hline 20 & 26 & FA & $11: 00$ axis, right breast & 3969 & 2 \\
\hline
\end{tabular}

$I D C$ invasive ductal carcinoma, $F A$ fibroadenoma, BI-RADS Breast Imaging Reporting and Data System 
a

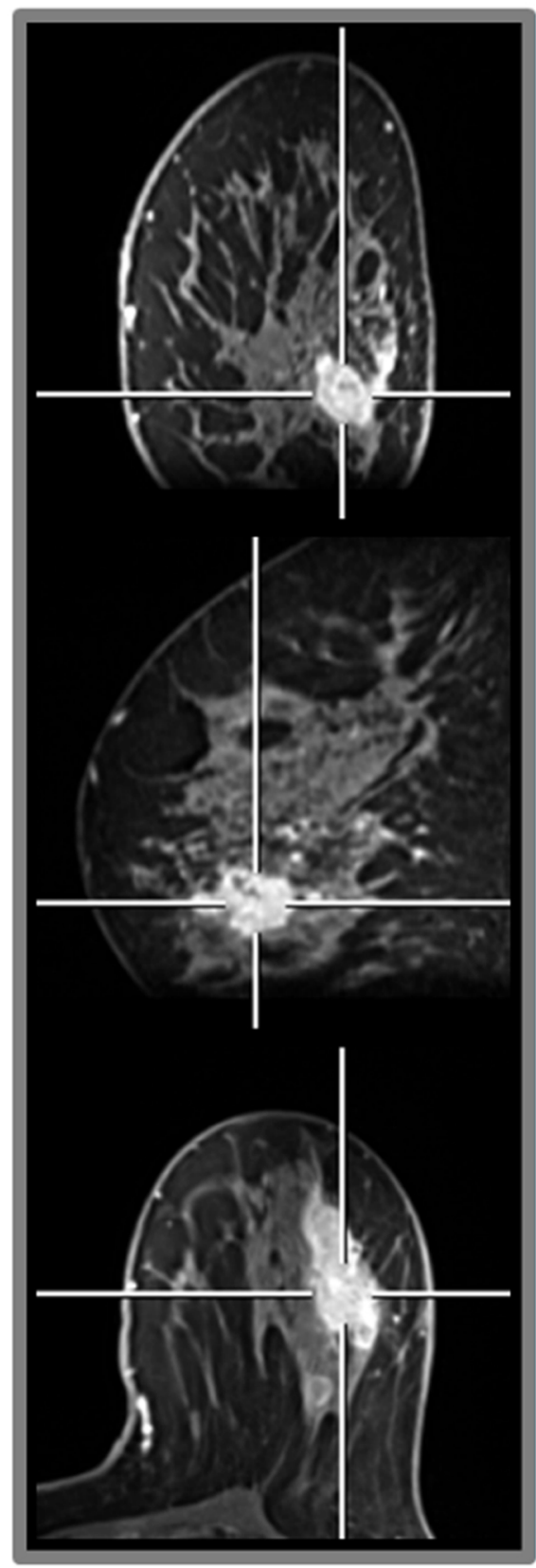

b Oxygen Metabolism

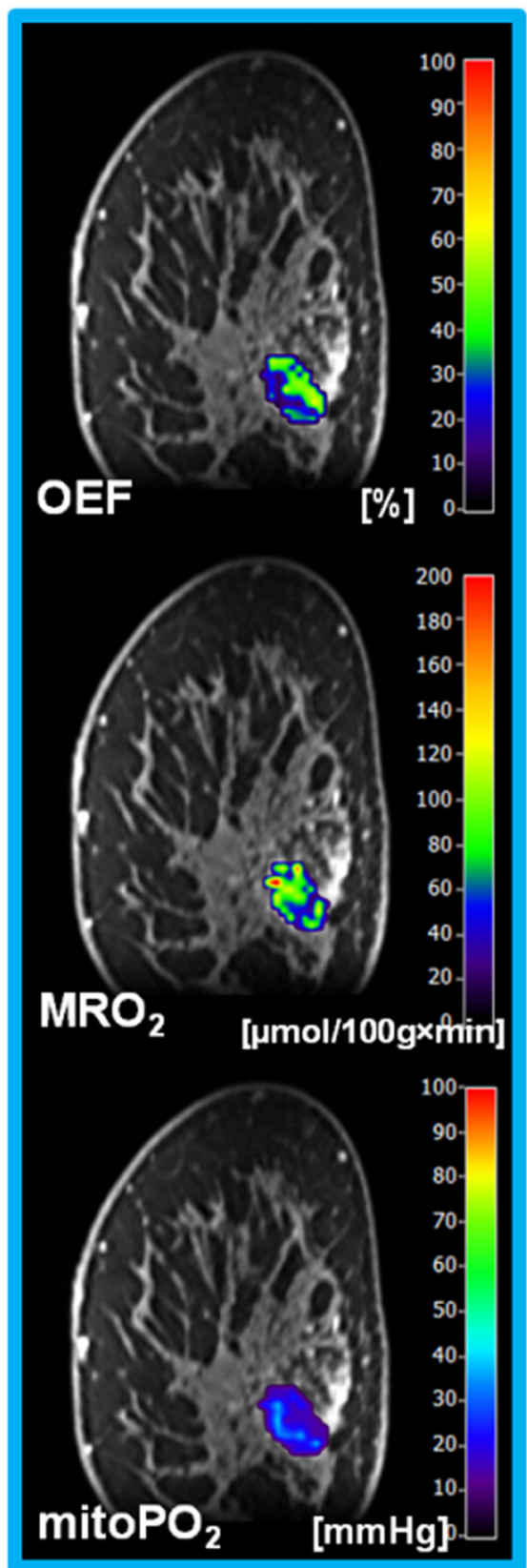

\section{Neovasculariztion}

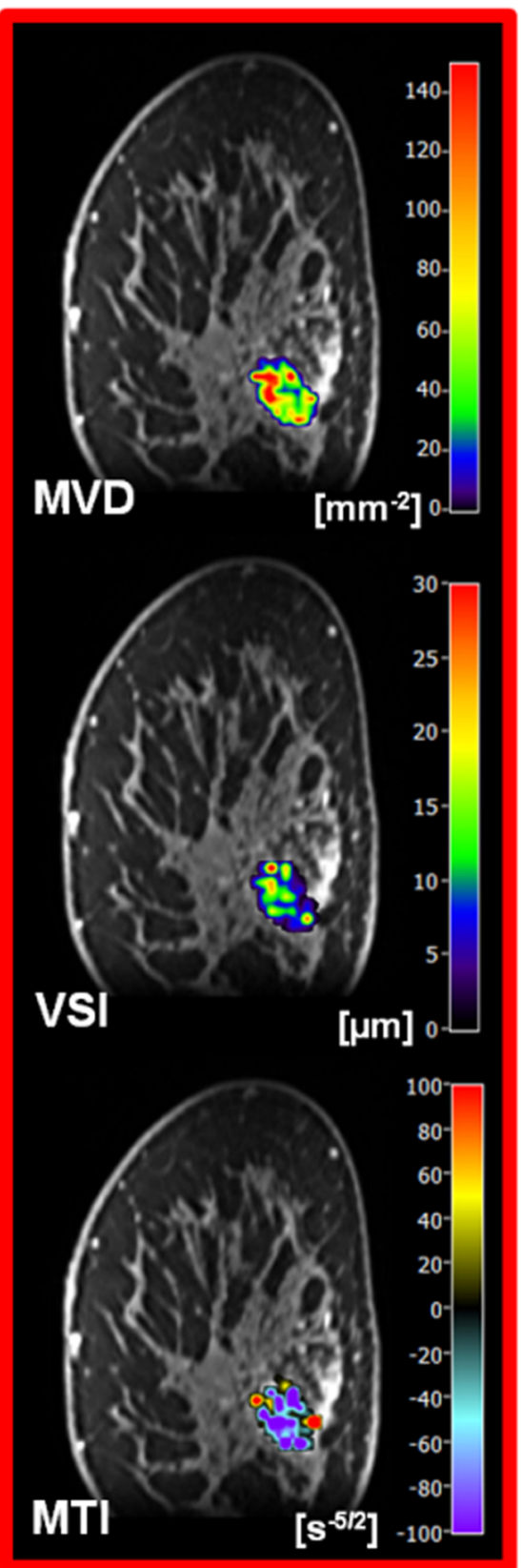

Fig. 2. Non-invasive synergistic assessment of neovascularization, oxygen metabolism, and hypoxia, respectively, in a 53year-old patient with an invasive ductal carcinoma (IDC, patient 14 in Table 1). a Conventional MRI (cMRI) using dynamic contrast-enhanced (DCE) T1-weighted perfusion MR images in coronal, sagittal, and axial orientation (top-down) show lesion size and position. The white line indicates the slice intersection. b Imaging biomarker maps of oxygen extraction fraction (OEF), metabolic rate of oxygen $\left(\mathrm{MRO}_{2}\right)$, and mitochondrial oxygen tension (mitoPO microvessel density (MVD), vessel size index (VSI), and micro vessel type indicator (MTI) in coronal orientation (top-down) demonstrates intratumoral spatial heterogeneity. The IDC showed high $\mathrm{MRO}_{2}$, low mitoPO , high MVD, and low (i.e., more pathologic) MTI.

demonstrated intratumoral spatial heterogeneity with a broad range of biomarker values. High OEF and low mitoPO $\mathrm{PO}_{2}$ (i.e., hypoxia) were associated with low MVD and vice versa. The heterogeneous pattern of $\mathrm{MRO}_{2}$ values, however, showed spatial congruence with VSI.
The MTI map revealed that the bulk of the tumor neovasculature consisted of draining venous microvasculature with slow flowing blood (purple regions). Additionally, the MTI map allowed for the detection of supplying arterial vessel structures which were 
identifiable as red and orange areas. These qualitative observations were confirmed by the remaining patients including those with benign lesions. A representative case of a patient with fibroadenoma is described in Fig. 3.

\section{Quantitative Analysis}

To investigate the intratumoral spatial heterogeneity of neovascularization, oxygen metabolism, and hypoxia, we performed an ROI-based quantitative analysis of the MRI a

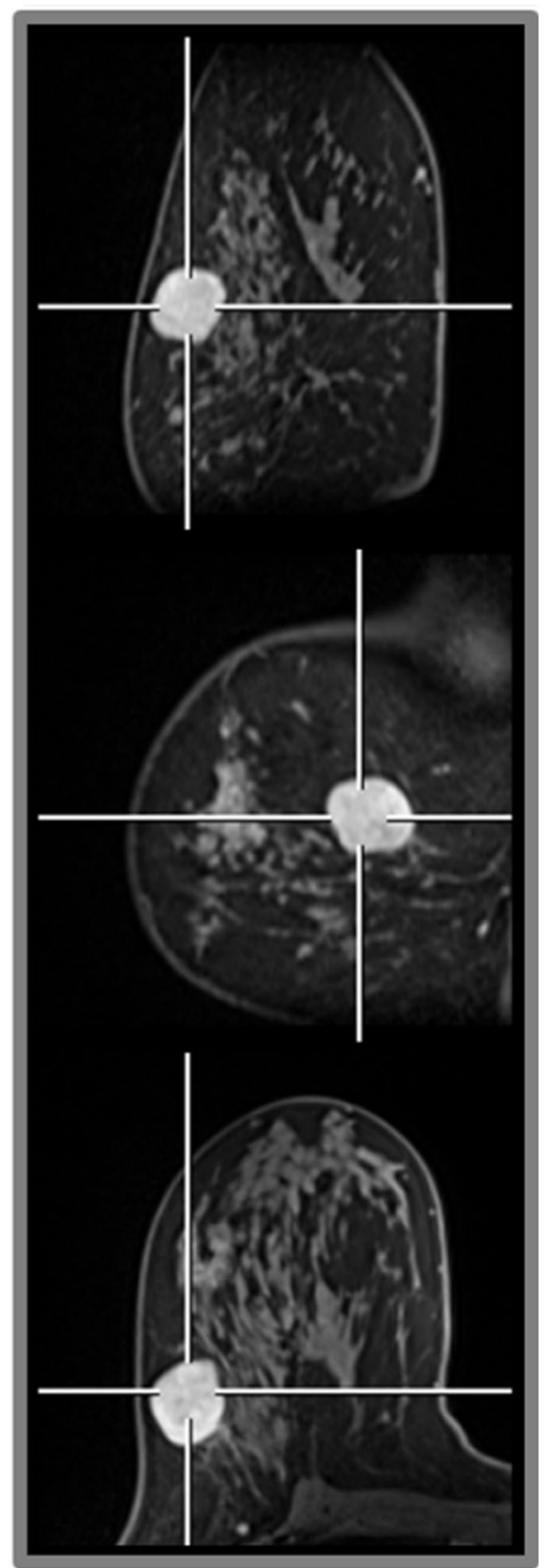

b Oxygen Metabolism

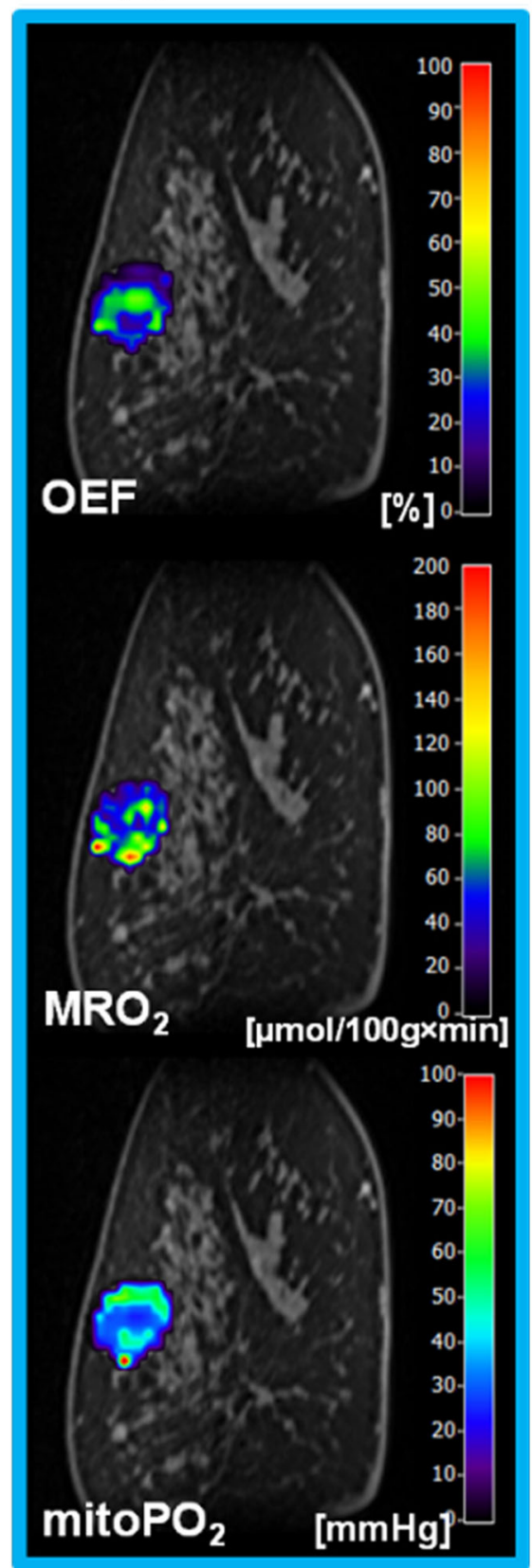

\section{Neovasculariztion}

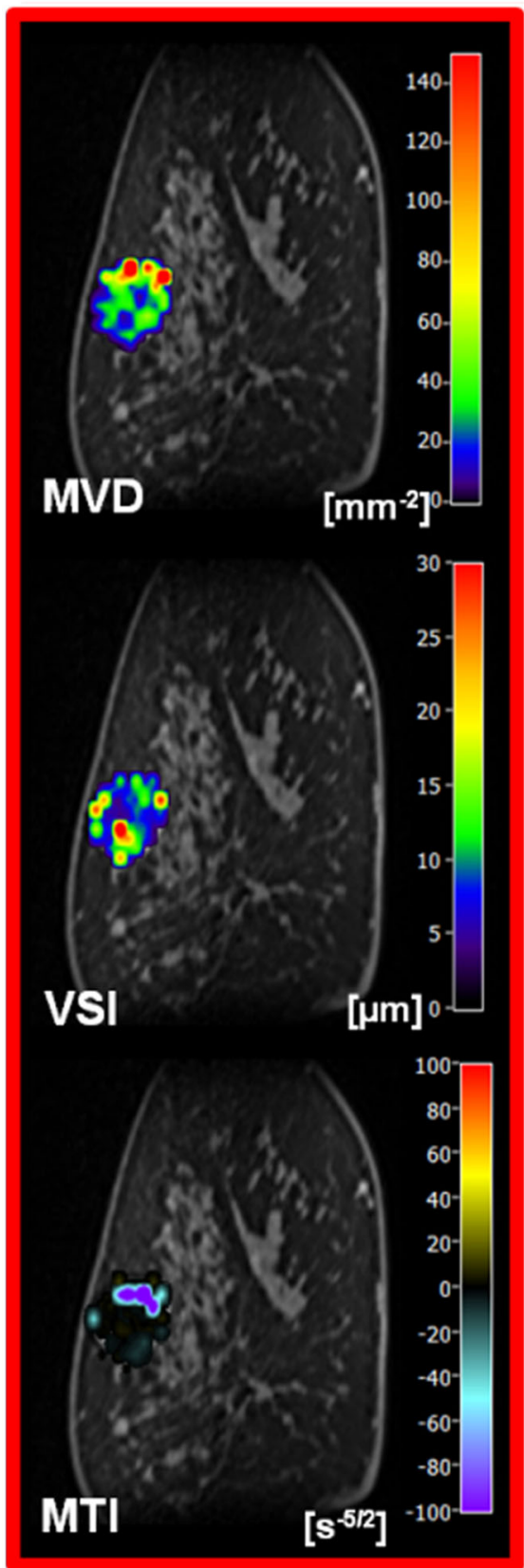

Fig. 3. Non-invasive synergistic assessment of neovascularization, oxygen metabolism, and hypoxia, respectively, in a 31year-old patient with a benign fibroadenoma (FA, patient 16 in Table 1). a Conventional MRI (cMRI) using dynamic contrastenhanced (DCE) T1-weighted perfusion MR images in coronal, sagittal, and axial orientation (top-down) show lesion size and position. The white line indicates the slice intersection. b Imaging biomarker maps of oxygen extraction fraction (OEF), metabolic rate of oxygen $\left(\mathrm{MRO}_{2}\right)$, and mitochondrial oxygen tension (mitoPO $\mathrm{P}_{2}$ ) in coronal orientation (top-down) as well as c microvessel density (MVD), vessel size index (VSI), and micro vessel type indicator (MTI) in coronal orientation (top-down) demonstrate intratumoral spatial heterogeneity with lower $\mathrm{MRO}_{2}$, higher mitoPO malignant tumors, e.g., Fig. 2. 
biomarker maps. For this purpose, ROIs were manually defined in areas showing high, moderate, and low neovascularization - as identified on the MVD maps - with high, moderate, and low MVD values, respectively. MVD were chosen for two reasons: (i) MVD is a common parameter for neovascularization, and (ii) it was demonstrated in a previous study that MVD is best suited for detection of neovascularization in brain tumors [26].

A histogram analysis of the MRI tumor hypoxia biomarkers through quantitative assessment of oxygen metabolism and neovascularization for benign (BI-RADS $2 / 3$ ) and malignant breast lesions (BI-RADS 5) is shown in Fig. 4. Breast carcinoma (IDC) showed significantly higher $\mathrm{MRO}_{2}(P=0.007$, Fig. 4b) and lower mitoPO $2(P=0.021$, Fig. 4c) as well as higher MVD $(P=0.005$, Fig. $4 d)$ and lower (i.e., more pathologic) MTI $(P=0.001$, Fig. 4f) compared with benign breast lesions. In other words, IDC lesions that consumed more oxygen were more hypoxic and neovascularized compared with benign lesions.

The results of correlation analyses between MRI biomarkers of neovascularization, oxygen metabolism, and hypoxia for benign and malign breast lesions, respectively, are shown in Fig. 5. In both benign and malignant breast lesions, OEF and MVD showed a significant negative correlation (BI-RADS 2/3: $R=-0.506, P=0.019$ and BIRADS 5: $R=-0.550, P<0.001)$ whereas $\mathrm{MRO}_{2}$ and VSI revealed a significant positive correlation (BI-RADS 2/3: $R=0.583, P=0.006$ and BI-RADS 5: $R=0.599, P<0.001$ ) with neovascularization, oxygen metabolism, and hypoxia, respectively (Fig. 5a, b, d, e).

In other words, the higher the MVD, the lower the necessary extraction of oxygen from the vasculature (i.e., OEF) to satisfy the oxygen demands. On the other hand, tumor regions with predominantly thicker microvessels (higher VSI) consumed more oxygen (higher $\mathrm{MRO}_{2}$ ). Furthermore, mitoPO $\mathrm{P}_{2}$ showed significantly positive correlation with MVD only for breast cancer (IDC; $R=0.514$, $P=0.001$; Fig. $5 \mathrm{f})$ but not for benign breast lesions $(R=$ $0.336, P=0.136$; Fig. 5c). Hence, the lower the MVD in IDC, the lower the oxygen tension in the tissue. All other correlations between MRI biomarkers for oxygen metabolism and neovascularization were not statistically significant.

\section{Discussion}

In breast cancer, tumor hypoxia is one of the key drivers of the development of an aggressive and treatmentresistant tumor phenotype and is a strong prognostic factor for disease progression, metastases, and survival [7-9]. In addition, several studies have reported an association between MVD and poorer recurrence-free, cancer-specific, and overall survival [11-13] as well as an association with clinical response to chemotherapy [14]. The distribution of hypoxia and induced neovascularization are not uniform within a tumor and therefore significantly contribute to $\mathrm{BC}$ heterogeneity [8].
To date, the assessment of tumor hypoxia, neovascularization, and $\mathrm{BC}$ heterogeneity is limited in their clinical application and there is a need for the development of an accurate, reliable, and non-invasive imaging technique that can assess tumor hypoxia, neovascularization, and BC heterogeneity to achieve improved tumor characterization and tailored treatment.

In this study, we developed a novel MRI approach for the non-invasive assessment of hypoxia and neovascularization in benign and malignant breast tumors. The MRI sequences can be easily integrated into a clinical routine MRI protocol, requiring less than $7 \mathrm{~min}$ of additional scan time and no additional injection of gadolinium-based contrast agents (GBCAs).

The proposed approach was tested in 20 consecutive patients with breast tumors and achieved good data quality in lesion with a diameter of at least $10 \mathrm{~mm}$ in all three spatial directions. MRI biomarker maps of neovascularization and oxygen metabolism for direct hypoxia measurement were successfully calculated.

Our approach demonstrated that breast cancer showed significantly higher $\mathrm{MRO}_{2}(P=0.007)$, lower mitoPO $\mathrm{PO}_{2}(P=$ $0.021)$, higher $\operatorname{MVD}(P=0.005)$, and lower (i.e., more pathologic) MTI $(P=0.001)$ compared with benign breast tumors. This indicates that breast cancer consumes more oxygen and is more hypoxic and neovascularized than benign tumors. These findings confirm the importance of tumor hypoxia and neovascularization as powerful physiological stimuli that can be exploited as a tumor-specific condition and can be used to design of hypoxia-based imaging biomarkers and hypoxia-activated anti-cancer drugs [40].

To date, only a few studies have used GE-based DSC perfusion sequences as part of a DCE MRI protocol. In most of these studies [41-44], two separate perfusion sequences in combination with two injections of contrast media resulting in up to a triple dose of contrast media were necessary. As it has been recently shown that the i.v. application of GBCAs may cause MRI signal changes in the brain, there is an ongoing debate regarding the safety and use of GBCAs. There is international consensus that the application of GBCAs should only be considered when necessary diagnostic information cannot otherwise be obtained and therefore administration of higher doses of the contrast agent are not acceptable within the recommendation [45-47].

In line with this recommendation, we developed a technique that allows consistent and direct measurement of neovascularization, oxygen metabolism, and hypoxia with the application of the single dose of GBCA already required in a standard imaging breast MRI protocol. This is a significant advancement over prior approaches.

Wang, et al. [48] developed a dual-echo GE sequence for simultaneous measurements of T1w DCE MRI and T2* $\mathrm{W}$ DSC MRI parameters in the breast that allowed the calculation of semi-quantitative parameters such as blood flow and blood volume. They showed that their approach 

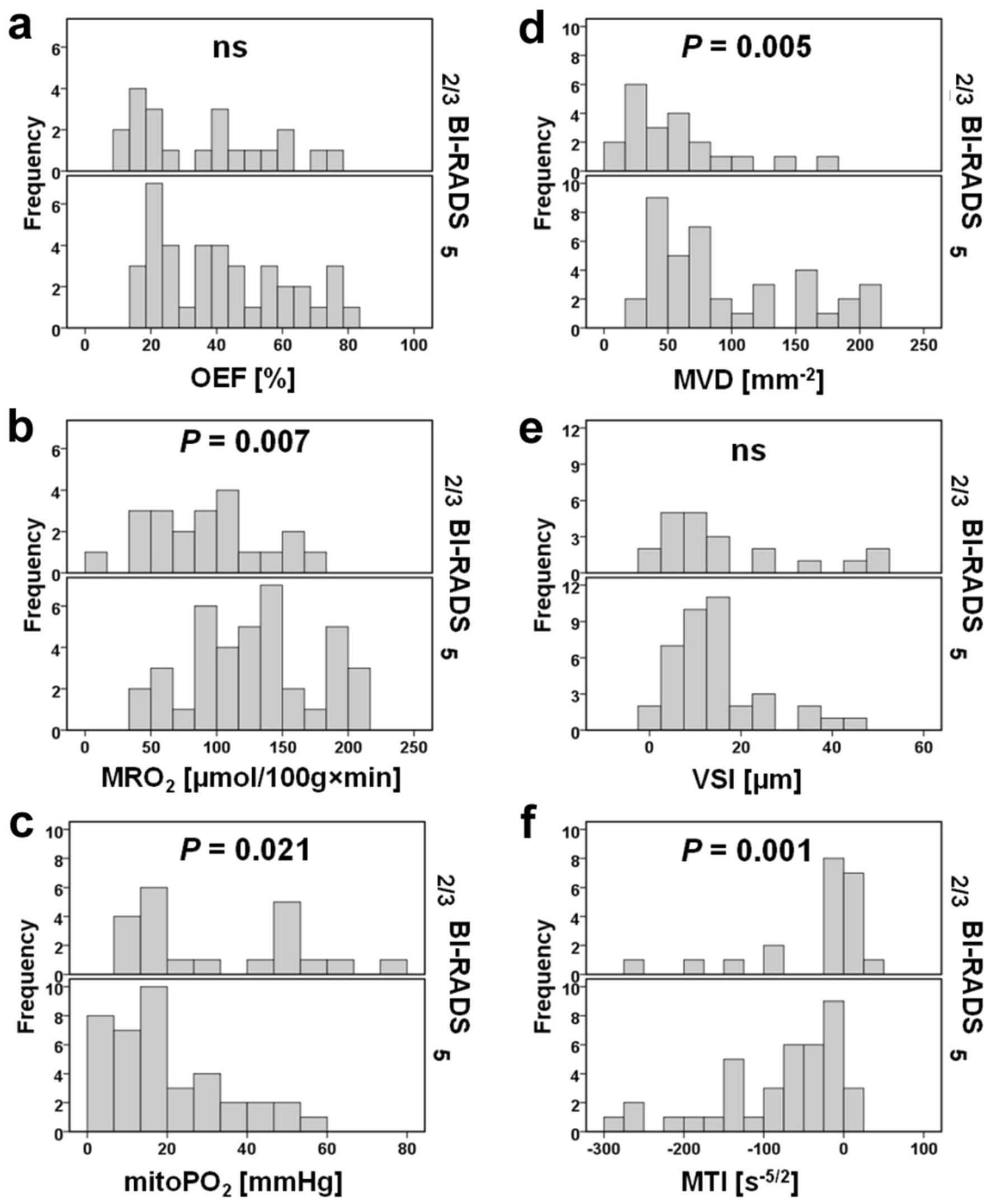

Fig. 4. Histogram analysis of a oxygen extraction fraction $(\mathrm{OEF})$, $\mathbf{b}$ metabolic rate of oxygen $\left(\mathrm{MRO}_{2}\right)$, $\mathbf{c}$ mitochondrial oxygen tension ( $\left(\mathrm{mitoPO}_{2}\right.$ ), $\mathbf{d}$ microvessel density (MVD), e vessel size index (VSI), and $\mathbf{f}$ microvessel type indicator (MTI) for benign (BIRADS 2/3) and malignant breast lesions (BI-RADS 5). $P$ values show significance of difference between the histograms as calculated with a Mann-Whitney test. 

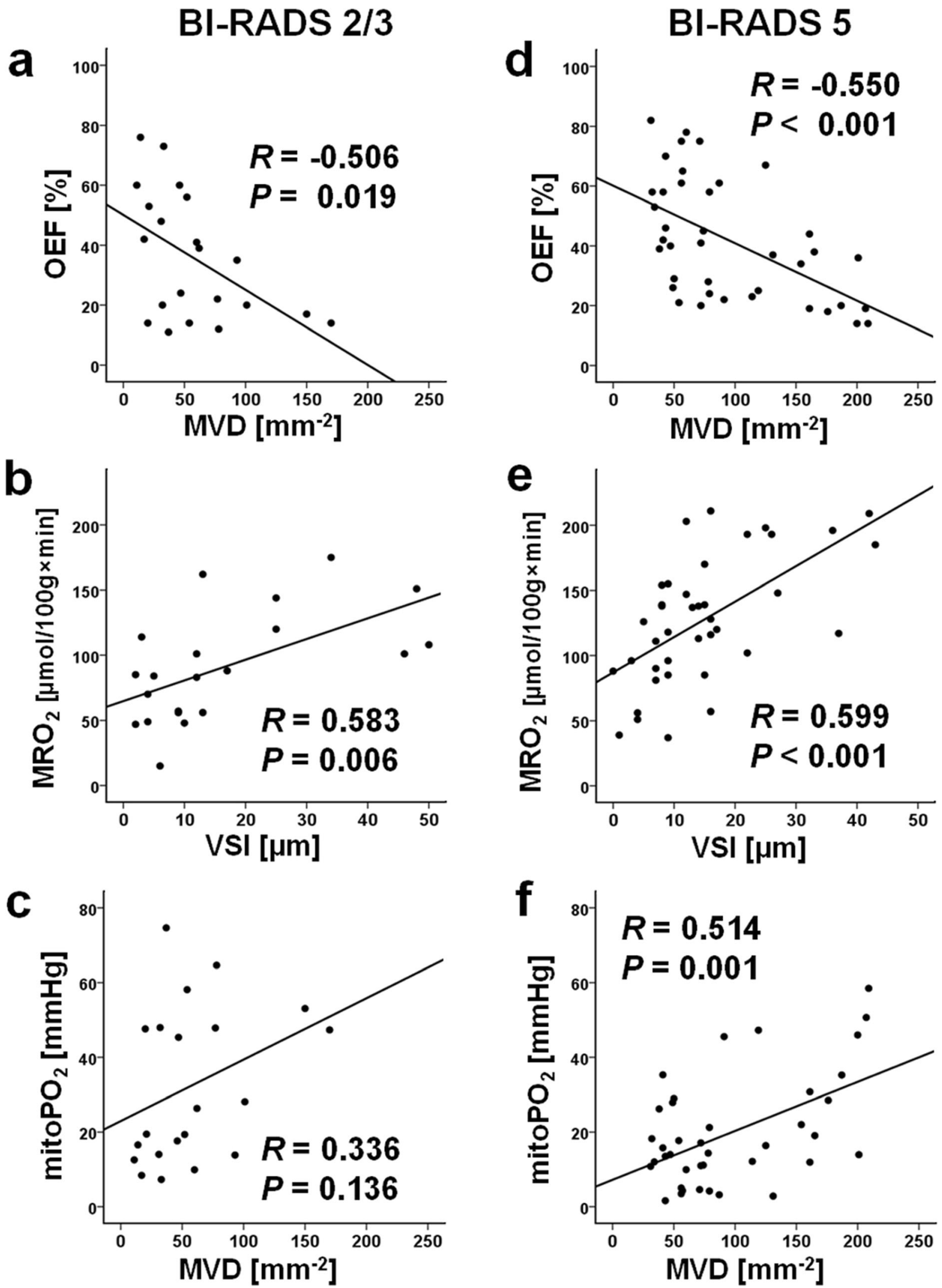

Fig. 5. Linear regression and Spearman's correlation analysis between MRI biomarker for neovascularization, oxygen metabolism, and hypoxia, respectively. Benign breast lesions (BI-RADS 2/3) revealed significant correlation between a extraction fraction (OEF) and microvessel density (MVD), and between $\mathbf{b}$ metabolic rate of oxygen $\left(\mathrm{MRO}_{2}\right)$ and vessel size index (VSI), but not between c mitochondrial oxygen tension (mitoPO ${ }_{2}$ ) and MVD. Breast cancer (BI-RADS 5), however, showed significant correlation between d OEF versus MVD, e $\mathrm{MRO}_{2}$ versus VSI, and $\mathbf{f}$ mitoPO ${ }_{2}$ versus $\mathrm{MVD}$, respectively. 
improved the diagnostic performance of breast MRI for the differentiation of breast cancer from benign fibroadenomas. However, in contrast to our synergistic approach, no information about microvascular architecture, i.e., MVD and VSI, was obtained as no SE-DSC perfusion MRI measurements were included. Kim et al. [49] used GE and SE steady-state susceptibility contrast MRI in an orthotopic human breast cancer xenograft model and computed MRI biomarker maps of MVD and VSI, which were validated with ex vivo whole tumor 3D micro-CT angiography. They found that the MRI biomarkers significantly correlated with their micro-CT analogs. The findings of the current study for MVD and VSI are in good agreement with these previous results. It has to be noted that none of the previous studies included examinations of oxygen metabolism and subsequently hypoxia with MRI or another technique. To date, invasive sampling with needle electrodes or PET using radioactive tracers such as ${ }^{15} \mathrm{O}-\mathrm{O}_{2}$ has been mainly used for the assessment of oxygen metabolism. Our results for tissue oxygen tension $\left(\right.$ mitoPO $\left.{ }_{2}\right)$ and $\mathrm{MRO}_{2}$ are in good agreement with these results [50-54] yet were obtained non-invasively and without ionizing radiation, which is an inherent benefit of our approach.

In breast cancer, tumor hypoxia has been recognized as an important driver of intratumoral heterogeneity which in turn leads to the development of cell clones with an aggressive and treatment-resistant phenotype characterized by rapid progression, treatment failures, and patient deaths $[1,2,6-9]$. Prior studies that investigated tumor hypoxia in breast cancer using the PET/CT imaging with the radiotracer $\left[{ }^{18} \mathrm{~F}\right]$ fluoromisonidazole $\left(\left[{ }^{18} \mathrm{~F}\right] \mathrm{FMISO}\right)$ have shown that higher $\left[{ }^{18} \mathrm{~F}\right] \mathrm{FMISO}$-tumor-to-background-ratio (TBR) and estrogen receptor negativity were independent predictors of shorter disease-free survival, where higher $\left[{ }^{18} \mathrm{~F}\right]$ FMISO-TBR was associated with higher plasma levels of angiogenic hypoxic markers [55]. Cheng, et al. also showed that $\left[{ }^{18} \mathrm{~F}\right]$ FMISO PET/CT can be used to predict primary endocrine resistance in ER-positive breast cancer [20]. A limitation of the assessment of tumor hypoxia with PET/CT is the associated radiation dose and long up-take and examination times. In addition, in comparison to MRI, the spatial resolution is much lower limiting the detailed depiction of intratumoral heterogeneity. In the current study, MRI biomarker maps of neovascularization, oxygen metabolism, and hypoxia, respectively, showed intratumoral spatial heterogeneity with a broad range of MRI biomarker values. We found that $\mathrm{OEF}$ inversely and mitoPO $\mathrm{P}_{2}$ directly correlated with MVD. This might be explained by the fact that in a less dense tumor microcirculation (low MVD), higher extraction of oxygen from the vasculature (high OEF) is necessary to satisfy the oxygen demands of the tumor. Additionally, the tissue oxygen tension is lower in these regions compared with areas with higher MVD. Consequently, neovascularization is initiated to sustain oxygen and nutrient supply and enable the further progression of the tumor. The further the development of the tumor microcirculation as driven by ongoing neovascularization, the higher the VSI and the possible oxygen consumption $\left(\mathrm{MRO}_{2}\right)$ of the tumor region. In this study, we expand on the current knowledge by providing non-invasive insight into the distinct intratumoral heterogeneity of breast cancer, which in the future may be used to develop hypoxia-based imaging biomarkers and guide targeted treatment with hypoxia-activated anticancer drugs.

A current limitation of the study is that due to the lower signal-to-noise ratio of the employed sequence, in particular regarding the SE-DSC data compared with a standard T1w DCE perfusion sequence, only tumors with a diameter of at least $10 \mathrm{~mm}$ in all three spatial directions were included. However, there is active research to improve spatial resolution of qBOLD and VAM imaging. It can therefore be expected that in the future, smaller lesions can also be assessed and this current limitation will be overcome. In this study, we performed no direct histopathologic correlation for MRI biomarkers. It has to be noted that previous studies that assessed MVD by immunohistochemistry of biopsy samples using antibodies against different factors such as VIII, CD31, and CD34 revealed very heterogeneous results with MVD ranging between less than 10 and few hundred $\mathrm{mm}^{-2}$ [56-58]. These discrepancies, apart from differences in MVD assessment methodology and patient selection criteria, might be explained by intratumoral heterogeneity which was demonstrated in this study. Therefore, histopathological evaluation of whole tumor sections precisely registered to the pre-operative MRI data which mandates sophisticated planning is required for validation and was beyond the scope of this study. The aim of this feasibility study was the development of the MRI approach, and direct histopathologic correlation with derived MRI biomarkers is part of an on-going larger-scale study.

\section{Conclusions}

In conclusion, we developed a novel MRI approach for the non-invasive assessment of hypoxia and neovascularization in benign and malignant breast tumors and provide insight into the distinct intratumoral heterogeneity of breast cancer. Our non-invasive approach can be easily integrated in a diagnostic MRI protocol and does not require additional application of GBCAs and has the potential to improve tumor characterization and facilitate tailored breast cancer treatment. However promising, the performance, and the fashion of implementation, of this novel MRI approach for the non-invasive assessment of hypoxia and neovascularization in benign and malignant breast tumors remains to be further investigated.

Acknowledgements. Open access funding provided by Medical University of Vienna.

Funding. This work was supported by the Anniversary Fund of the Oesterreichische Nationalbank (OeNB; project number 16219). Katja Pinker 
was also supported by the National Institutes of Health/National Cancer Institute Cancer Center Support Grant P30 CA008748.

Compliance with Ethical Standards. The institutional review board approved this prospective, single-institution study, and all patients gave written, informed consent.

\section{Conflict of Interest}

The authors declare that they have no conflict of interest.

\section{Ethical Approval}

All procedures performed in studies involving human participants were in accordance with the ethical standards of the institutional and/or national research committee and with the 1964 Helsinki declaration and its later amendments or comparable ethical standards.

\section{Informed Consent}

Informed consent was obtained from all individual participants included in the study.

Open Access This article is distributed under the terms of the Creative Commons Attribution 4.0 International License (http:// creativecommons.org/licenses/by/4.0/), which permits unrestricted use, distribution, and reproduction in any medium, provided you give appropriate credit to the original author(s) and the source, provide a link to the Creative Commons license, and indicate if changes were made.

\section{References}

1. Zardavas D, Irrthum A, Swanton C, Piccart M (2015) Clinical management of breast cancer heterogeneity. Nat Rev Clin Oncol 12:381-394

2. Haynes B, Sarma A, Nangia-Makker P, Shekhar MP (2017) Breast cancer complexity: implications of intratumoral heterogeneity in clinical management. Cancer Metastasis Rev 36:547-555

3. Wirapati P, Sotiriou C, Kunkel S, Farmer P, Pradervand S, HaibeKains B, Desmedt C, Ignatiadis M, Sengstag T, Schütz F, Goldstein DR, Piccart M, Delorenzi M (2008) Meta-analysis of gene expression profiles in breast cancer: toward a unified understanding of breast cancer subtyping and prognosis signatures. Breast Cancer Res: BCR 10:R65

4. Lam SW, Jimenez CR, Boven E (2014) Breast cancer classification by proteomic technologies: current state of knowledge. Cancer Treat Rev 40:129-138

5. Huber KE, Carey LA, Wazer DE (2009) Breast cancer molecular subtypes in patients with locally advanced disease: impact on prognosis, patterns of recurrence, and response to therapy. Semin Radiat Oncol 19:204-210

6. Martelotto LG, Ng CK, Piscuoglio S et al (2014) Breast cancer intratumor heterogeneity. Breast Cancer Res: BCR 16:210

7. Ruan K, Song G, Ouyang G (2009) Role of hypoxia in the hallmarks of human cancer. J Cell Biochem 107:1053-1062

8. Vaupel P (2008) Hypoxia and aggressive tumor phenotype: implications for therapy and prognosis. Oncologist 13(Suppl 3):21-26

9. Hockel M, Schlenger K, Mitze M et al (1996) Hypoxia and radiation response in human tumors. Semin Radiat Oncol 6:3-9

10. Hanahan D, Weinberg RA (2011) Hallmarks of cancer: the next generation. Cell 144:646-674

11. Tsutsui S, Kume M, Era S (2003) Prognostic value of microvessel density in invasive ductal carcinoma of the breast. Breast Cancer (Tokyo, Japan) 10:312-319

12. Bevilacqua P, Barbareschi M, Verderio P, Boracchi P, Caffo O, Palma PD, Meli S, Weidner N, Gasparini G (1995) Prognostic value of intratumoral microvessel density, a measure of tumor angiogenesis, in node-negative breast carcinoma-results of a multiparametric study. Breast Cancer Res Treat 36:205-217

13. Al Murri AM, Wilson C, Lannigan A et al (2007) Evaluation of the relationship between the systemic inflammatory response and cancerspecific survival in patients with primary operable breast cancer. Br J Cancer 96:891-895
14. Foekens JA, Peters HA, Grebenchtchikov N et al (2001) High tumor levels of vascular endothelial growth factor predict poor response to systemic therapy in advanced breast cancer. Cancer Res 61:5407-5414

15. van Brussel AS, Adams A, Vermeulen JF et al (2013) Molecular imaging with a fluorescent antibody targeting carbonic anhydrase IX can successfully detect hypoxic ductal carcinoma in situ of the breast. Breast Cancer Res Treat 140:263-272

16. Goddard JC, Sutton CD, Furness PN, Kockelbergh RC, O'Byrne KJ (2002) A computer image analysis system for microvessel density measurement in solid tumours. Angiogenesis 5:15-20

17. Uzzan B, Nicolas P, Cucherat M, Perret GY (2004) Microvessel density as a prognostic factor in women with breast cancer: a systematic review of the literature and meta-analysis. Cancer Res 64:2941-2955

18. Rajendran JG, Mankoff DA, O'Sullivan F et al (2004) Hypoxia and glucose metabolism in malignant tumors: evaluation by $\left[{ }^{18} \mathrm{~F}\right]$ fluoromisonidazole and $\left[{ }^{18} \mathrm{~F}\right]$ fluorodeoxyglucose positron emission tomography imaging. Clin Cancer Res 10:2245-2252

19. Ueda S, Saeki T, Osaki A, Yamane T, Kuji I (2017) Bevacizumab induces acute hypoxia and Cancer progression in patients with refractory breast Cancer: multimodal functional imaging and multiplex cytokine analysis. Clin Cancer Res 23:5769-5778

20. Cheng J, Lei L, Xu J, Sun Y, Zhang Y, Wang X, Pan L, Shao Z, Zhang Y, Liu G (2013) ${ }^{18}$ F-fluoromisonidazole PET/CT: a potential tool for predicting primary endocrine therapy resistance in breast cancer. J Nucl Med 54:333-340

21. Yu Y, Zhang K, Zhang L et al (2018) Cerebral near-infrared spectroscopy (NIRS) for perioperative monitoring of brain oxygenation in children and adults. Cochrane Database Syst Rev 1:CD010947

22. Hyttel-Sorensen S, Pellicer A, Alderliesten T et al (2015) Cerebral near infrared spectroscopy oximetry in extremely preterm infants: phase II randomised clinical trial. BMJ g7635:350

23. Davies DJ, Clancy M, Dehghani H, Lucas SJE, Forcione M, Yakoub KM, Belli A (2018) Cerebral oxygenation in traumatic brain injury: can a non-invasive frequency domain near-infrared spectroscopy device detect changes in brain tissue oxygen tension as well as the established invasive monitor? J Neurotrauma. https://doi.org/10.1089/ neu. 2018.5667

24. Stadlbauer A, Zimmermann M, Heinz G, Oberndorfer S, Doerfler A, Buchfelder M, Rössler K (2017) Magnetic resonance imaging biomarkers for clinical routine assessment of microvascular architecture in glioma. J Cereb Blood Flow Metab 37:632-643

25. Stadlbauer A, Mouridsen K, Doerfler A, Bo Hansen M, Oberndorfer S, Zimmermann M, Buchfelder M, Heinz G, Roessler K (2018) Recurrence of glioblastoma is associated with elevated microvascular transit time heterogeneity and increased hypoxia. J Cereb Blood Flow Metab 38:422-432

26. Stadlbauer A, Zimmermann M, Kitzwogerer M et al (2017) MR imaging-derived oxygen metabolism and neovascularization characterization for grading and IDH gene mutation detection of gliomas. Radiology 283:799-809

27. Christen T, Schmiedeskamp H, Straka M, Bammer R, Zaharchuk G (2012) Measuring brain oxygenation in humans using a multiparametric quantitative blood oxygenation level dependent MRI approach. Magn Reson Med 68:905-911

28. Gjedde A, Johannsen P, Cold GE, Ostergaard L (2005) Cerebral metabolic response to low blood flow: possible role of cytochrome oxidase inhibition. J Cereb Blood Flow Metab 25:1183-1196

29. Stadlbauer A, Zimmermann M, Oberndorfer S, Doerfler A, Buchfelder M, Heinz G, Roessler K (2017) Vascular hysteresis loops and vascular architecture mapping in patients with glioblastoma treated with antiangiogenic therapy. Sci Rep 7:8508

30. Mann RM, Balleyguier C, Baltzer PA et al (2015) Breast MRI: EUSOBI recommendations for women's information. Eur Radiol 25:3669-3678

31. Xu C, Kiselev VG, Moller HE, Fiebach JB (2013) Dynamic hysteresis between gradient echo and spin echo attenuations in dynamic susceptibility contrast imaging. Magn Reson Med 69:981-991

32. Preibisch C, Volz S, Anti S, Deichmann R (2008) Exponential excitation pulses for improved water content mapping in the presence of background gradients. Magn Reson Med 60:908-916

33. Prasloski T, Madler B, Xiang QS et al (2012) Applications of stimulated echo correction to multicomponent T2 analysis. Magn Reson Med 67:1803-1814 
34. Delille JP, Slanetz PJ, Yeh ED, Kopans DB, Garrido L (2002) Breast cancer: regional blood flow and blood volume measured with magnetic susceptibility-based MR imaging-initial results. Radiology 223:558-565

35. Gjedde A (2002) Cerebral blood flow change in arterial hypoxemia is consistent with negligible oxygen tension in brain mitochondria. NeuroImage 17:1876-1881

36. Vafaee MS, Vang K, Bergersen LH, Gjedde A (2012) Oxygen consumption and blood flow coupling in human motor cortex during intense finger tapping: implication for a role of lactate. J Cereb Blood Flow Metabol 32:1859-1868

37. Ducreux D, Buvat I, Meder JF et al (2006) Perfusion-weighted MR imaging studies in brain hypervascular diseases: comparison of arterial input function extractions for perfusion measurement. Am J Neuroradiol 27:1059-1069

38. Jensen JH, Lu H, Inglese M (2006) Microvessel density estimation in the human brain by means of dynamic contrast-enhanced echo-planar imaging. Magn Reson Med 56:1145-1150

39. Emblem KE, Mouridsen K, Bjornerud A, Farrar CT, Jennings D, Borra RJH, Wen PY, Ivy P, Batchelor TT, Rosen BR, Jain RK, Sorensen AG (2013) Vessel architectural imaging identifies cancer patient responders to anti-angiogenic therapy. Nature Med 19:11781183

40. Vaupel P, Mayer A (2015) The clinical importance of assessing tumor hypoxia: relationship of tumor hypoxia to prognosis and therapeutic opportunities. Antioxid Redox Signal 22:878-880

41. Kvistad KA, Rydland J, Vainio J, Smethurst HB, Lundgren S, Fjøsne HE, Haraldseth O (2000) Breast lesions: evaluation with dynamic contrast-enhanced T1-weighted MR imaging and with T2*-weighted first-pass perfusion MR imaging. Radiology 216:545-553

42. Dannert S, Kruck W, Schick F et al (2001) MR tomographic characterization of suspicious breast lesions with dynamic susceptibility-weighted T2* sequences. RöFo 173:38-43

43. Li SP, Taylor NJ, Makris A, Ah-See MLW, Beresford MJ, Stirling JJ, d'Arcy JA, Collins DJ, Padhani AR (2010) Primary human breast adenocarcinoma: imaging and histologic correlates of intrinsic susceptibility-weighted MR imaging before and during chemotherapy. Radiology 257:643-652

44. Li SP, Padhani AR, Taylor NJ, Beresford MJ, Ah-See MLW, Stirling JJ, d'Arcy JA, Collins DJ, Makris A (2011) Vascular characterisation of triple negative breast carcinomas using dynamic MRI. Eur Radiol 21:1364-1373

45. Runge VM (2016) Safety of the gadolinium-based contrast agents for magnetic resonance imaging, focusing in part on their accumulation in the brain and especially the dentate nucleus. Investig Radiol 51:273279

46. Gulani V, Calamante F, Shellock FG, Kanal E, Reeder SB, International Society for Magnetic Resonance in M (2017)
Gadolinium deposition in the brain: summary of evidence and recommendations. Lancet Neurol 16:564-570

47. Runge VM (2017) Critical questions regarding gadolinium deposition in the brain and body after injections of the gadolinium-based contrast agents, safety, and clinical recommendations in consideration of the EMA's pharmacovigilance and risk assessment committee recommendation for suspension of the marketing authorizations for 4 linear agents. Investig Radiol 52:317-323

48. Wang S, Delproposto Z, Wang H et al (2013) Differentiation of breast cancer from fibroadenoma with dual-echo dynamic contrast-enhanced MRI. PLoS One 8:e67731

49. Kim E, Cebulla J, Ward BD, Rhie K, Zhang J, Pathak AP (2013) Assessing breast cancer angiogenesis in vivo: which susceptibility contrast MRI biomarkers are relevant? Magn Reson Med 70:11061116

50. Hohenberger P, Felgner C, Haensch W, Schlag PM (1998) Tumor oxygenation correlates with molecular growth determinants in breast cancer. Breast Cancer Res Treat 48:97-106

51. Semenza GL (2016) The hypoxic tumor microenvironment: a driving force for breast cancer progression. Biochim Biophys Acta 1863:382391

52. Vaupel P, Schlenger K, Knoop C, Hockel M (1991) Oxygenation of human tumors: evaluation of tissue oxygen distribution in breast cancers by computerized $\mathrm{O} 2$ tension measurements. Cancer Res 51:3316-3322

53. Grimes DR, Kannan P, McIntyre A, Kavanagh A, Siddiky A, Wigfield S, Harris A, Partridge M (2016) The role of oxygen in avascular tumor growth. PLoS One 11:e0153692

54. Kallinowski F, Schlenger KH, Kloes M, Stohrer M, Vaupel P (1989) Tumor blood flow: the principal modulator of oxidative and glycolytic metabolism, and of the metabolic micromilieu of human tumor xenografts in vivo. Int J Cancer 44:266-272

55. Asano A, Ueda S, Kuji I, Yamane T, Takeuchi H, Hirokawa E, Sugitani I, Shimada H, Hasebe T, Osaki A, Saeki T (2018) Intracellular hypoxia measured by (18)F-fluoromisonidazole positron emission tomography has prognostic impact in patients with estrogen receptor-positive breast cancer. Breast Cancer Res: BCR 20:78

56. Luczynska E, Niemiec J, Ambicka A et al (2015) Correlation between blood and lymphatic vessel density and results of contrast-enhanced spectral mammography. Pol J Pathol 66:310-322

57. Kruger K, Stefansson IM, Collett K, Arnes JB, Aas T, Akslen LA (2013) Microvessel proliferation by co-expression of endothelial nestin and $\mathrm{Ki}-67$ is associated with a basal-like phenotype and aggressive features in breast cancer. Breast (Edinburgh, Scotland) 22:282-288

58. Arnes JB, Stefansson IM, Straume O, Baak JP, Lønning PE, Foulkes WD, Akslen LA (2012) Vascular proliferation is a prognostic factor in breast cancer. Breast Cancer Res Tr 133:501-510 